\title{
Taurine Ameliorates Impaired the Mitochondrial Function and Prevents Stroke-like Episodes in Patients with MELAS
}

\author{
Mitsue Rikimaru ${ }^{1}$, Yutaka Ohsawa ${ }^{1}$, Alexander M. Wolf ${ }^{2}$, Kiyomi Nishimaki ${ }^{2}$, \\ Harumi Ichimiya ${ }^{2}$, Naomi Kamimura ${ }^{2}$, Shin-ichiro Nishimatsu ${ }^{3}$, \\ Shigeo Ohta ${ }^{2}$ and Yoshihide Sunada ${ }^{1}$
}

\begin{abstract}
Objective Post-transcriptional taurine modification at the first anticodon ("wobble") nucleotide is deficient in A3243G-mutant mitochondrial (mt) tRNA ${ }^{\mathrm{Leu}(\mathrm{UU} \mathrm{R})}$ of patients with myopathy, encephalopathy, lactic acidosis, and stroke-like episodes (MELAS). Wobble nucleotide modifications in tRNAs have recently been identified to be important in the accurate and efficient deciphering of codons. We herein examined whether taurine can alleviate mitochondrial dysfunction in patient-derived pathogenic cells and prevent clinical symptoms in MELAS patients.

Methods and Results The addition of taurine to the culture media ameliorated the reduced oxygen consumption, decreased the mitochondrial membrane potential, and increased the oxidative stress in MELAS patient-derived cells. Moreover, high dose oral administration of taurine $(0.25 \mathrm{~g} / \mathrm{kg} / \mathrm{day})$ completely prevented stroke-like episodes in two MELAS patients for more than nine years.

Conclusion Taurine supplementation may be a novel potential treatment option for preventing the strokelike episodes associated with MELAS.
\end{abstract}

Key words: MELAS, post-transcriptional modification, taurine, stroke-like episodes

(Intern Med 51: 3351-3357, 2012)

(DOI: 10.2169/internalmedicine.51.7529)

\section{Introduction}

An A3243G or T3271C transition in the mitochondrial (mt) tRNA $^{\text {Leu(UUR) }}$ gene has been identified in approximately $80 \%$ and $10 \%$ respectively, of patients with mitochondrial myopathy, encephalopathy, lactic acidosis, and stroke-like episodes (MELAS) (1). Nearly 90\% of patients with myoclonus epilepsy associated with ragged-red fibers (MERRF) possess an A8344G transition in the $m t \mathrm{tRNA}^{\mathrm{Lys}}$ gene (1). These mutations are located in the cloverleaf structure of each mt tRNA. However, it remains unknown how such point mutations in mt tRNAs induce mitochondrial dysfunction leading to the wide variety of MELAS or MERRF symptoms.

Post-transcriptional modifications in tRNAs play critical roles in modifying the genetic code. In 1966, Francis Crick proposed that the first anticodon ("wobble") nucleotide recognizes the third codon nucleotide through non-canonical Watson-Crick geometry; so-called "wobble" pairing (2). Growing evidence has shown that various posttranscriptional modifications at the wobble nucleotides in tRNAs are required to recognize their cognate codons accurately and efficiently (3). In normal human mt tRNA ${ }^{\text {Leu(UUR) }}$ or $\mathrm{mt} \mathrm{tRNA}^{\mathrm{Lys}}$, uridine at the wobble position is modified with taurine, a sulfur-containing $\beta$-amino acid (4-6). In contrast, the taurine modification is deficient in mutant $\mathrm{mt}$ tRNA $^{\text {Leu(UUR) }}$ or mutant $m t$ tRNA ${ }^{\text {Lys }}$ derived from clinical specimens of MELAS or MERRF patients (4-8). The taurine modification defect in the mutant mt tRNAs causes a deficiency in deciphering codons $(1,9)$. These findings have given rise to the intriguing possibility that MELAS and

${ }^{1}$ Department of Neurology, Kawasaki Medical School, Japan, ${ }^{2}$ Department of Biochemistry and Cell Biology, Institute of Development and Aging Sciences, Nippon Medical School, Japan and ${ }^{3}$ Department of Molecular and Developmental Biology, Kawasaki Medical School, Japan Received for publication February 13, 2012; Accepted for publication August 6, 2012

Correspondence to Dr. Yoshihide Sunada, ysunada@med.kawasaki-m.ac.jp 
MERRF are tRNA-modification disorders associated with the impairment of correct mitochondrial gene translation.

We hypothesized that high-dose taurine supplementation could restore the taurine modification of the mutant tRNAs in MELAS or MERRF patients. In the current study, we explored the potential therapeutic effect of taurine by examining the mitochondrial functions in patient-derived pathogenic cells and by observing the clinical symptoms in MELAS patients receiving taurine supplements.

\section{Materials and Methods}

The local ethics committee approved this study (No. 787) and all patients gave their informed consent for participation.

\section{Construction of cybrid cells harboring mutant mtDNA}

Immortalized cells possessing patient-derived mitochondrial (mt) DNA were constructed by the intercellular transfer of a patient's mtDNA to $\rho^{0} \mathrm{HeLa}$ cells (EB8), which are mtDNA-less immortalized cells (10). EB8 cells were isolated by the long-term treatment of HeLa cells with ethidium bromide. Primary dermal fibroblasts were isolated from skin biopsy samples from an A3243G-MELAS, a T3271CMELAS, and an A8344G-MERRF patient. The fibroblasts were enucleated by centrifugation in the presence of cytochalasin B. Then, the enucleated fibroblasts were fused with EB8 cells by treatment with polyethylene glycol. Control cytoplasmic hybrid (cybrid) strains (Ft2-11, A2) were constructed by fusing mtDNA-less HeLa cells with enucleated normal human fibroblasts.

The resulting cybrids were maintained in Dulbecco's modified Eagle's medium/Ham's F12 medium supplemented with $10 \%$ fetal bovine serum, $1 \mathrm{mM}$ sodium pyruvate, $50 \mu \mathrm{g}$ uridine, 100 units $/ \mathrm{mL}$ penicillin, and $100 \mu \mathrm{g} / \mathrm{mL}$ streptomycin (Invitrogen/Life Technologies Japan, Tokyo, Japan). Cybrids with more than $95 \%$ mutant mtDNA were used for the experiments. To decrease the endogenous taurine, the cells were also cultured in media with limited amounts of the taurine precursor, L-cysteine $(1 \mathrm{mg} / \mathrm{mL})$, and the taurine intermediate, L-methionine (high glucose, L-glutamine-minus, sodium pyruvate-minus Dulbecco's modified Eagle's medium; Gibco) supplemented with L-glutamine, sodium pyruvate, and uridine. The growth rate of mutant cybrids was unchanged after culture in limiting media for seven days.

\section{Cell lines and in vitro analyses}

Primary dermal fibroblasts obtained from skin biopsy samples from an A3243G-MELAS, a T3271C-MELAS, and an A8344G-MERRF patient were enucleated and subsequently fused with mt DNA-less HeLa cells (10). The resulting cybrid cells were treated with or without taurine and then were used in subsequent in vitro analyses of the mitochondrial oxygen consumption (11), membrane potential (12), and reduction and oxidation (redox) status (10).
Taurine powder was purchased from Taisho Pharmaceutical Co., Ltd. (Tokyo, Japan).

\section{Mitochondrial oxygen consumption}

Cybrid cells cultured with or without taurine were trypsinized and resuspended in serum-free medium. The cell suspension was continuously stirred at $37^{\circ} \mathrm{C}$ with an oxygen electrode (11). The cell concentration was determined using a hemocytometer. The oxygen consumption rates were measured using an Oxygen Meter Model 781 and a Mitocell MT200 closed respiratory chamber (Strathkelvin Instruments, North Lanarkshire, UK). The oxygen respiration rate was directly measured for the $40 \mathrm{mM}$ taurine experiments. After treatment with the limiting media described above, the oxygen consumption was examined in the presence of $1 \mu \mathrm{M}$ carbonyl cyanide p-trifluoromethoxyphenylhydrazone (FCCP), a mitochondrial protonophore used to measure electron transport activity. The consumption value was subtracted from the $1 \mathrm{mM}$ potassium cyanide-independent oxygen consumption value.

\section{Mitochondrial membrane potential}

To evaluate the mitochondrial membrane potential, cybrid cells were incubated for 30 minutes at $37^{\circ} \mathrm{C}$ with $20 \mathrm{nM}$ MitoTracker Red (Molecular Probes, Invitrogen, Carlsbad, CA, USA), a red-fluorescent dye that accumulates at the mitochondrial membrane (12) in response to the membrane potential. The MitoTracker Red signal increases in a membrane potential-dependent manner. The images were visualized with a confocal laser-scanning microscope (Fluoview FV300; Olympus, Tokyo, Japan) at an excitation wavelength of $594 \mathrm{~nm}$. For the flow cytometric analysis, cells stained with MitoTracker Red were washed in phosphate-buffered saline, trypsinized, and analyzed using a Cell Lab Quanta ${ }^{\mathrm{TM}}$ instrument (Beckman Coulter, Inc., Brea, CA, USA). The fluorescent signal of more than 10,000 cells was examined for each experiment.

\section{Mitochondrial redox status}

The redox-sensitive green fluorescent protein, roGFP1, generates a unique fluorescence image after the formation (oxidation) of the disulfide bonds adjacent to the barreled $\beta$ sheets in the GFP protein (11). To allow real-time visualization of mitochondrial redox status, cybrid cells were stably transfected with the roGFP1 expression vector containing a mitochondrial-targeting sequence. Fluorescence images were recorded using a multi-dimensional imaging workstation (AS MDW; Leica Microsystems, Wetzlar, Germany) consisting of a tunable light source (Polychrome IV monochromator; Till Photonics, Gräfelfing, Germany), an inverted epifluorescence microscope (DM IRE2; Leica Microsystems) contained in a climate chamber maintained at $37^{\circ} \mathrm{C}$, and a cooled charge-coupled device camera (CoolSnap HQ; Roper Scientific, Princeton, NJ, USA). The dual excitation ratio of roGFP1 from a single cell was recorded. The ratio of the reduced form of roGFP1 (roGFP1-SH) to the oxidized form 
A

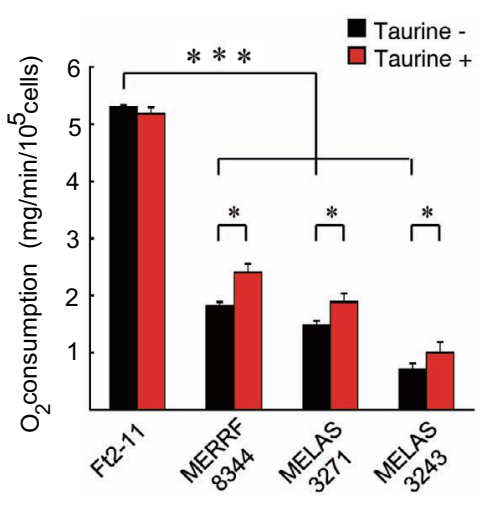

B

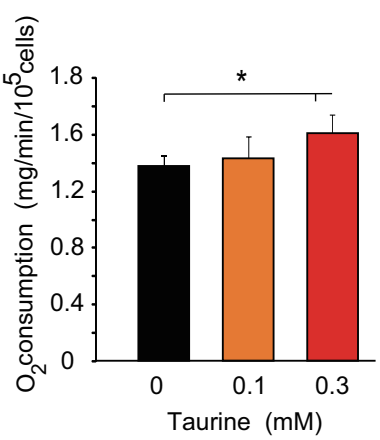

C
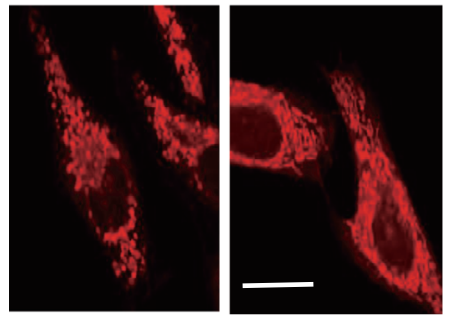

D

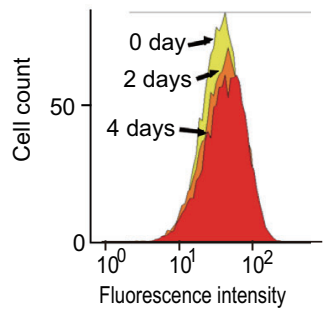

E
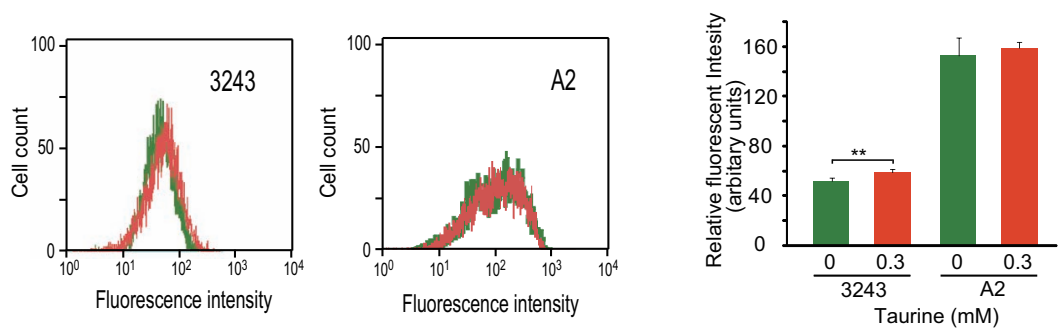

Figure 1. Taurine ameliorates the impaired mitochondrial function in patient-derived cybrid cells. (A) Patient-derived cybrid cells showed marked decreases in oxygen consumption (black bars). After four days in culture with taurine $(40 \mathrm{mM})$, there was a significant increase in the oxygen consumption rates in patient-derived cybrids with mutant mtDNA, but not in wild-type control Ft2-11 cells (red bars) $(* \mathbf{p}<0.05)$. (B) Cybrids were cultured in media with limited amounts of the taurine intermediate, L-methionine $(1 \mathrm{mg} / \mathrm{mL})$, and the taurine precursor, L-cysteine $(5 \mathrm{mg} / \mathrm{mL})$, for two days, followed by an additional four day culture with or without taurine $(0,0.1$, or $0.3 \mathrm{mM})$. Taurine ( $0.3 \mathrm{mM}$ ) improved the oxygen consumption in the A3243G-MELAS cybrids cultured in the limiting media (*p < 0.05). (C) Cybrids were cultured in the presence (right) or absence (left) of $40 \mathrm{mM}$ taurine for 4 days. Staining with the membrane potential-sensitive red-fluorescent dye MitoTracker Red $(100 \mathrm{nM}$ for $30 \mathrm{~min})$ revealed an increased mitochondrial membrane potential with morphological improvement in the A3243G-MELAS cybrid cells. Scale bar: $100 \mu \mathrm{m}$. (D) The mitochondrial membrane potential was determined by a flow cytometric analysis after staining with $100 \mathrm{nM}$ of MitoTracker Red for $30 \mathrm{~min}$. The profiles in the left-hand panel show a time-dependent increase in membrane potential after incubation with $40 \mathrm{mM}$ taurine. The right-hand profiles indicate that there was a dose-dependent shift in the membrane potential after four days of culture with the indicated amounts of taurine. (E) Cybrid cells were cultured in the limiting media described in (B). The reduced mitochondrial membrane potential in the A3243G-MELAS cybrid cells (3243) was significantly improved as judged by a flow cytometric analysis after a four-day incubation with $0.3 \mathrm{mM}$ taurine (*p $<0.05$ ). In contrast, the membrane potential in the control cybrid cells (A2) was unchanged after taurine treatment. 

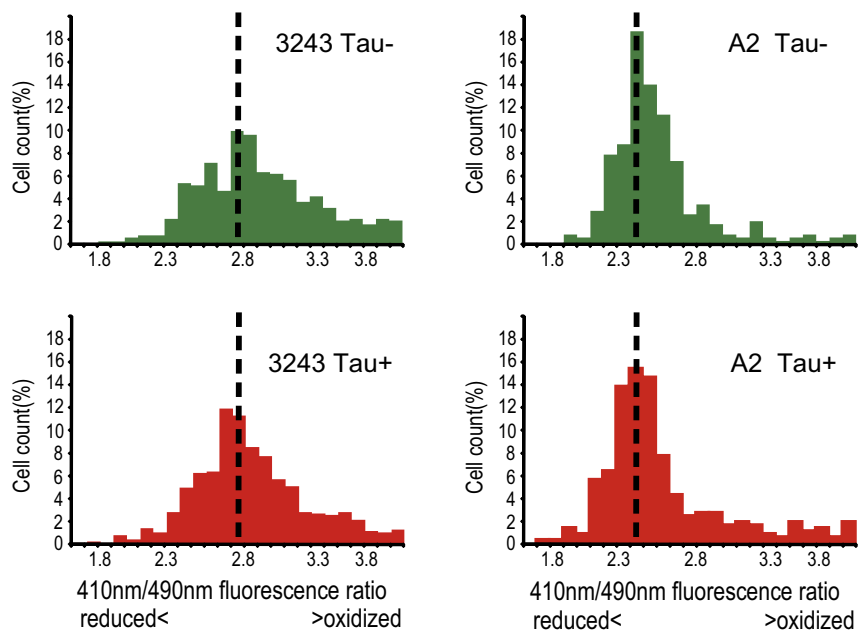

Figure 2. Taurine reduces the oxidative stress in patient-derived cybrid cells. The A3243G-MELAS cybrid cells (3243, left) and the control cybrid cells (A2, right) were stably transfected with a mitochondria-targeting redox-sensitive green fluorescent protein (roGFP). The histograms show the distribution of cells according to their $410: 490 \mathrm{~nm}$ fluorescence ratio, an indicator of the oxidation status. Compared with the A2 cybrid cells, the ratio was increased in the 3243 cybrid cells, suggesting an increase in oxidative stress (green, upper) The addition of taurine ( $3 \mathrm{mM}$; red, lower) caused a shift towards a reduced status in the 3243 cybrid cells, but not in the A2 cybrid cells (red, lower). The data represent the mean values from eight independent experiments. *p $<0.05$ between culture conditions with and without taurine.

\section{Oral administration of taurine to patients with A3243G-MELAS}

Taurine powder was orally administered three times a day, after a meal, to two patients with A3243G-MELAS at a dose of $0.25 \mathrm{~g} / \mathrm{kg} / \mathrm{day}$. This corresponds to the maximal dose previously employed for Japanese patients with biliary obstructions (13).

\section{Statistical analyses}

Paired observations were statistically analyzed using a one-way analysis of variance followed by Bonferroni's test. $\mathrm{p}$ values $<0.05$ were considered to be statistically significant.

\section{Results}

\section{Taurine restores the reduced mitochondrial oxygen consumption in patient-derived cells}

The cybrid cells harboring the disease-causing mutant mtDNAs showed lower oxygen consumption rates than the control cells (Fig. 1A). The addition of $40 \mathrm{mM}$ taurine to the culture media increased the oxygen consumption rate in patient-derived cybrid cells, but not in control cells. Moreover, $0.3 \mathrm{mM}$ taurine was also effective when the cybrid cells were cultured in limiting media lacking cysteine and methionine, which are a precursor and an intermediate, respectively, of taurine biogenesis (Fig. 1B).

\section{Taurine improves the reduced mitochondrial mem- brane potential in A3243G-MELAS cells}

MitoTracker Red-labeled mitochondria in the A3243GMELAS cybrid cells displayed a weak signal with a granular appearance, suggesting that they had a decreased mitochondrial membrane potential compared to normal cells (Fig. 1C, left) (12). When the cybrid cells were cultivated in the presence of $40 \mathrm{mM}$ taurine for four days, the mitochondria underwent changes in their morphology to a normal filamentous appearance, which was accompanied by an increase in the membrane potential (Fig. 1C, right) (12). The reduced mitochondrial membrane potential in the A3243G-MELAS cells was reversed by taurine in a timeand concentration-dependent manner (Fig. 1D). Moreover, $0.3 \mathrm{mM}$ taurine increased the membrane potential in the A3243G-MELAS cybrids that were cultured in limiting media (Fig. 1E). In contrast, taurine did not alter the membrane potential in the control A2 cybrid cells.

\section{Taurine improves the impaired redox status in patient-derived cells}

We transfected the MELAS-cybrid cells with a gene encoding a redox-sensitive green fluorescent protein, roGFP, to monitor their redox status as judged by the ratio of fluorescence signals at 410 and $490 \mathrm{~nm}$ (11). The ratio in the A3243G-MELAS cybrid cells increased in comparison to that in the control cells, thus suggesting that they had an increased degree of oxidative stress (Fig. 2, upper). The addition of taurine to the culture media reduced the ratio in the A3243G-MELAS cybrid cells, but not in the control cells (Fig. 2, lower).

\section{Taurine prevents stroke-like episodes in A3243G- MELAS patients}

Case 1: A 29-year-old woman had an abrupt onset of generalized seizures and was admitted to our hospital in February 2001 (Fig. 3A). The lactate and pyruvate levels in her serum were elevated to $48.3 \mathrm{mg} / \mathrm{dL}$ (normal range, 3.0-17.0 $\mathrm{mg} / \mathrm{dL}$ ) and $1.7 \mathrm{mg} / \mathrm{dL}$ (normal range, $0.3-0.9 \mathrm{mg} / \mathrm{dL}$ ), respectively. Brain magnetic resonance imaging (MRI) revealed a stroke-like lesion in the left occipital region (Fig. 3B). A biopsy from the left biceps brachii muscle showed a MELAS-like pattern, with cytochrome $\mathrm{c}$ oxidasenegative ragged-red fibers and succinate dehydrogenasereactive blood vessels. A molecular genetic analysis of the mitochondrial DNA confirmed an A3243G transition. Treatment with coenzyme Q10 (180 mg daily) and phenytoin (250 mg daily) was commenced in February 2001. The anticonvulsant was switched from phenytoin to valproate $(600$ mg daily) in April 2001 because of repeated generalized seizures. A follow-up MRI in August 2001 revealed an additional right occipitotemporal lesion (Fig. 3C). The patient continued to experience epileptic seizures and had a stroke- 
A

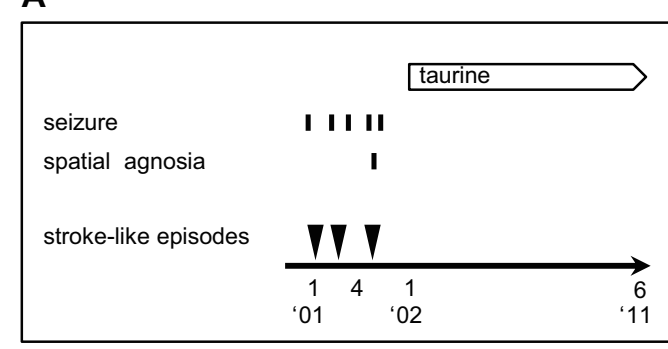

E

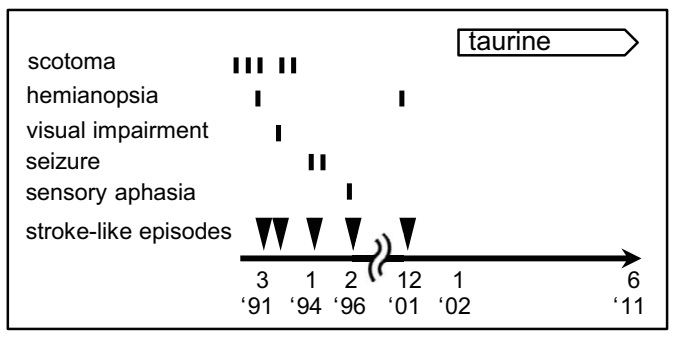

B

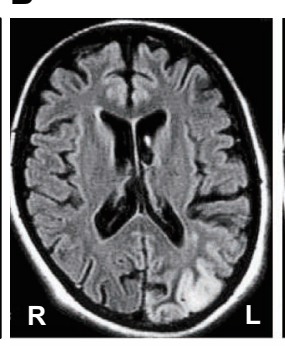

$\mathbf{F}$

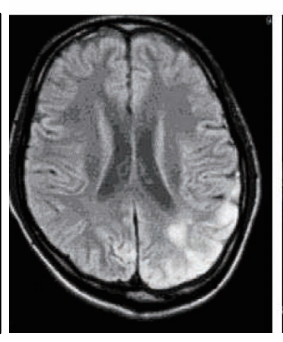

C

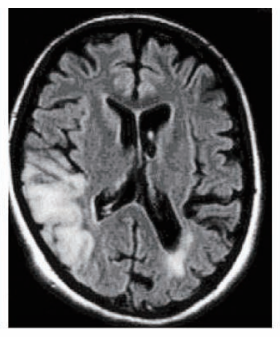

G

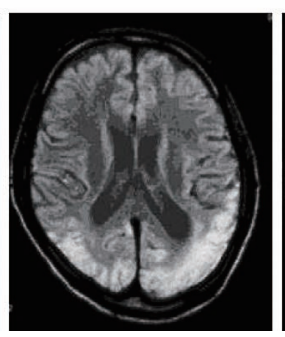

D

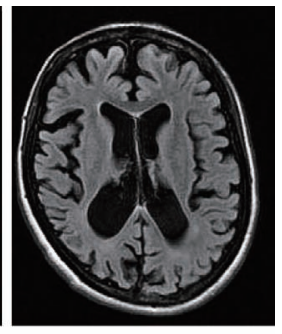

H

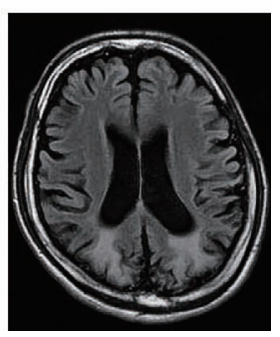

Figure 3. Oral administration of taurine reduces the stroke-like episodes in MELAS patients. The clinical courses of two MELAS patients (Cases 1 and 2) harboring the A3243G mutation in the mt tRNA $^{\text {Leu(UUR) }}(A, E)$ are shown. Taurine administration completely prevented stroke-like episodes in both patients for more than nine years. Fluid Attenuated Inversion Recovery (FLAIR) images of brain MRI revealed that multiple stroke-like lesions had developed in the occipitotemporal region before oral taurine administration (B, C, F, G). The most recent MRI showed no additional strokelike lesions after taurine treatment in both patients $(\mathrm{D}, \mathrm{H})$.

like episode presenting hemispatial agnosia over the next seven months. Oral taurine supplementation was started in January 2002. From the beginning of the taurine treatment, her epileptic and stroke-like episodes ceased completely. In July 2007, her blood concentration of taurine was $481.3 \mu \mathrm{M}$, more than 5-fold higher than the normal range (39.5-93.2 $\mu \mathrm{M})$. In December 2010, the elevated levels of serum lactate and pyruvate had declined to near normal levels, at $24.3 \mathrm{mg} /$ $\mathrm{dL}$ and $0.9 \mathrm{mg} / \mathrm{dL}$, respectively. The most recent brain MRI exhibited no new lesions, but mild cerebral atrophy was present (Fig. 3D). The patient has been doing well for the last nine years with the taurine treatment still ongoing.

Case 2: A 21-year-old man was admitted to another hospital in March 1991 because of repeated scintillating scotoma and right homonymous hemianopsia (Fig. 3E). He was diagnosed with A3243G-MELAS based on typical muscle biopsy findings and a mtDNA analysis. He was treated with coenzyme Q10 (120 mg/dL) and phenytoin (150 mg daily); however, he soon developed vision loss on the right side. He was admitted to our hospital in July 1991. The serum levels of lactate and pyruvate were elevated to $38.7 \mathrm{mg} / \mathrm{dL}$ and 1.2 $\mathrm{mg} / \mathrm{dL}$, respectively. The anticonvulsant was switched from phenytoin to valproate (600 mg daily) in January 1994 because of repeated generalized seizures. Over the next eight years he suffered from several stroke-like episodes, including sensory aphasia and visual impairment. Brain MRI scans in October 1991 and January 1994 revealed an accumulation of stroke lesions in the bilateral occipital regions (Fig. 3F, G). In December 2001 he had a stroke-like episode presenting with left hemianopsia. Taurine supplementation was started in January 2002, and since then, no stroke-like episodes have occurred. In September 2007, his blood taurine concentration was $996.0 \mu \mathrm{M}$, approximately 10-fold higher than the normal range. In February 2010, the serum values of lactate and pyruvate had declined to $29.1 \mathrm{mg} / \mathrm{dL}$ and $0.38 \mathrm{mg} / \mathrm{dL}$, respectively. The most recent brain MRI exhibited no additional stroke-like lesions (Fig. 3H).

\section{Discussion}

Post-transcriptional modifications at the wobble nucleotide are crucial for the maturation mechanisms of tRNAs and they are required for the correct decoding of codons. In A3243G-MELAS patients, the taurine modification is defective at the wobble nucleotide in the mutant $\mathrm{mt}$ tRNA $^{\text {Leu(UUR) }}$ (5). In the present study, we showed that taurine ameliorates the mitochondrial dysfunction in patient-derived pathogenic cells carrying mutant tRNA ${ }^{\mathrm{Leu(UUR})}$, but did not reinforce the normal mitochondrial function in control cells. Oral taurine administration also achieved long-term prevention of stroke-like episodes in two patients with MELAS.

We previously showed that when taurine $(\tau)$ is added to the culture media of HeLa cells, it is transported to the mitochondria and used as a substrate to synthesize taurinemodified uridine, 5-taunomethyluridine $\left(\tau \mathrm{m}^{5} \mathrm{U}\right)$, in $\mathrm{mt}$ tRNA $^{\text {Leu(UUR) }}$ (Fig. 4A) (1, 4-7). Considering that $\tau \mathrm{m}^{5} \mathrm{U}$ formation proceeds through an enzymatic reaction, the present results suggest that an increased concentration of taurine ac- 
A

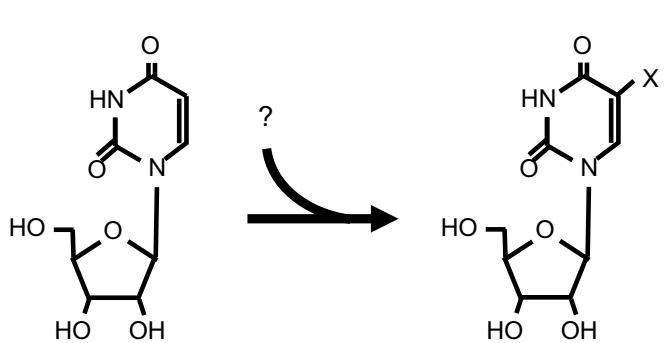

Uridine, $U$

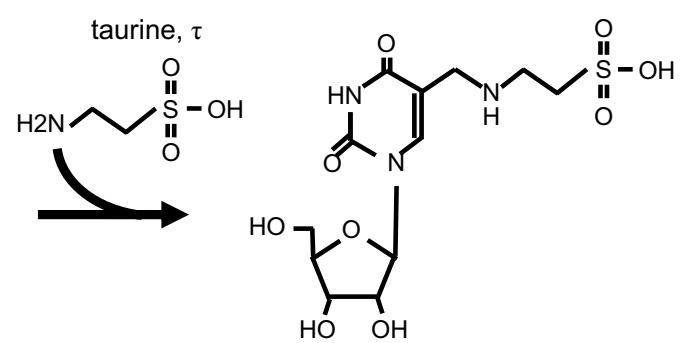

5'-taurinomethyl uridine, $\tau \mathrm{m}^{5} \mathrm{U}$

B

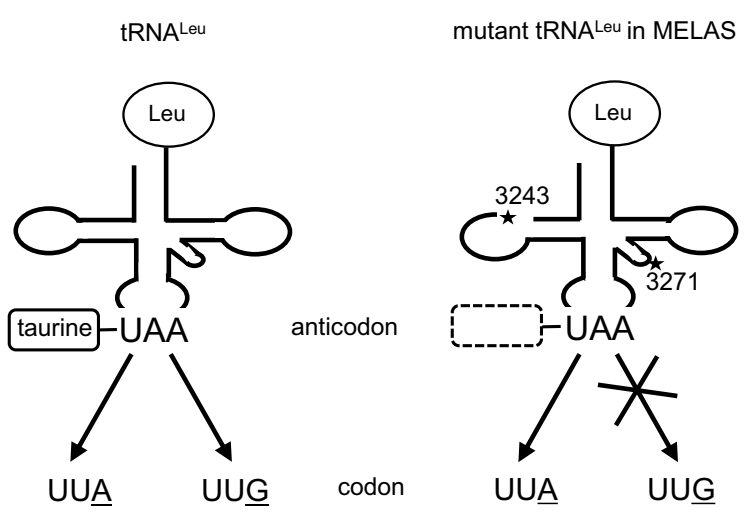

Figure 4. A proposed pathomechanism of MELAS, an RNA-modification disorder. (A) A mechanism of post-transcriptional taurine modification at the first wobble anticodon [uridine (U)] in normal $\mathrm{mt} \mathrm{tRNA}^{\mathrm{Leu}(\mathrm{UUR})}$. Taurine $(\tau)$ is incorporated into the $\mathrm{C5}$ position of the uracil ring to generate the final modification product, 5-taurinomethyluridine $\left(\tau \mathbf{m}^{5} \mathrm{U}\right)(4)$. (B) Taurine modification func-

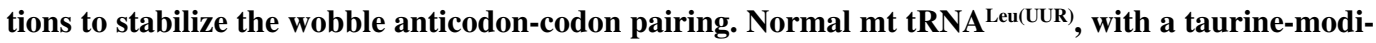

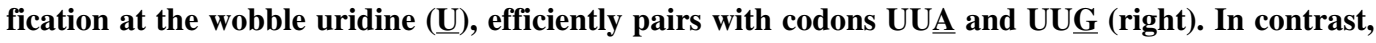
the MELAS-causing mutant $\mathrm{mt}$ tRNA ${ }^{\mathrm{Leu}(U U R)}$ lacks the wobble taurine modification, resulting in a specific reduction of UUG codon-specific translation but not UUA codon-specific translation. Defective taurine modification in the mutant $m t$ tRNA ${ }^{\mathrm{Leu}(U \mathrm{UR})}$ results in a deficiency in mitochondrial protein synthesis caused by an inability to decipher codons (left) (7).

celerates the enzymatic formation of $\tau \mathrm{m}^{5} \mathrm{U}$, thereby reversing impaired codon recognition by the mutant $\mathrm{mt}$ tRNA $^{\text {Leu(UUR) }}$ (Fig. 4B). The pathogenic mutations in MELAS and MERRF might hinder the specific recognition by an RNA-modifying enzyme (4-7). Further studies will be required to clarify the precise molecular mechanisms underlying the wobble taurine modification in $\mathrm{mt}_{\mathrm{tRNA}}{ }^{\mathrm{Leu(UUR})}$, and how much supplemented taurine incorporates into the wobble uridine in mutant $\mathrm{mt}_{\mathrm{tRNA}}^{\mathrm{Len(UUR)}}$ in clinical samples from patients.

Low plasma concentrations of taurine induce cardiomyopathy in cats. This particular species has no biosynthetic pathway for endogenous taurine (14). In agreement with our results, high-dose oral administration of taurine to cats increased the plasma and cardiac concentrations, and ameliorated the cardiac dysfunction. Because the cardiac muscles are composed of slow myofibers that are rich in mitochondria (14), taurine supplementation could alleviate the cardiomyopathy via increased $\tau \mathrm{m}^{5} \mathrm{U}$ formation in $\mathrm{mt}$ tRNAs.

The present results provide new insight into our under- standing of MELAS, and possibly MERRF, as putative RNA-modification disorders that lack the wobble taurine modification. Our results also suggest that the oral administration of taurine may be an effective therapy for these disorders.

The authors state that they have no Conflict of Interest (COI).

\section{Acknowledgement}

This work was supported by research grants for Intramural Neurological and Psychiatric Disorders from the National Center of Neurology and Psychiatry (20B-13, 23-5); by grants for Comprehensive Research on Disability Health and Welfare from the Ministry of Health, Labour and Welfare of Japan (H20-018); by Grants-in-Aid for Scientific Research from the Japan Society for the Promotion of Science (C-20591013, C-21591101, and C23591261); and by research project grants from the Kawasaki Medical School (22-A24, 21-T1, 23-P601, 23-T1). 


\section{References}

1. Suzuki T, Nagao A, Suzuki T. Human mitochondrial diseases caused by lack of taurine modification in mitochondrial tRNAs. WIREs RNA 2: 376-386, 2011.

2. Click FHC. Codon-anticodon pairing: the wobble hypothesis. J Mol Biol 19: 548-555, 1966.

3. Curran JF. Modified nucleosides in translation. In: Modification and Editing of RNA. Grosjean H, Benne R, Eds. ASM Press, Washington, D.C, 1998: 493-516.

4. Yasukawa T, Suzuki T, Ishii N, Ueda T, Ohta S, Watanabe K. Defect in modification at the anticodon wobble nucleotide of mitochondrial tRNA(Lys) with the MERRF encephalomyopathy pathogenic mutation. FEBS Lett 467: 175-178, 2000.

5. Yasukawa T, Suzuki T, Ueda T, Ohta S, Watanabe K. Modification defect at anticodon wobble nucleotide of mitochondrial tRNAs (Leu)(UUR) with pathogenic mutations of mitochondrial myopathy, encephalopathy, lactic acidosis, and stroke-like episodes. J Biol Chem 275: 4251-4257, 2000.

6. Yasukawa T, Suzuki T, Ishii N, Ohta S, Watanabe K. Wobble modification defect in tRNA disturbs codon-anticodon interaction in a mitochondrial disease. EMBO J 20: 4794-4802, 2001.

7. Suzuki T, Nagao A, Suzuki T. Human mitochondrial tRNAs: biogenesis, function, structural aspects, and diseases. Annu Rev Genet 45: 299-329, 2011.
8. Kirino Y, Goto Y, Campos Y, et al. Specific correlation between the wobble modification deficiency in mutant tRNAs and the clinical features of a human mitochondrial disease. Proc Natl Acad Sci USA 102: 7127-7132, 2005.

9. Kirino Y, Yasukawa T, Ohta S, et al. Codon-specific translational defect caused by a wobble modification deficiency in mutant tRNA from a human mitochondrial disease. Proc Natl Acad Sci USA 101: 15070-15075, 2004.

10. Hayashi J, Ohta S, Kikuchi A, et al. Introduction of diseaserelated mitochondrial DNA deletions into HeLa cells lacking mitochondrial DNA results in mitochondrial dysfunction. Proc Natl Acad Sci USA 88: 10614-10618, 1991.

11. Wolf AM, Asoh S, Hiranuma H, et al. Astaxanthin protects mitochondrial redox state and functional integrity against oxidative stress. J Nutr Biochem 21: 381-389, 2010.

12. Minamikawa T, Sriratana A, Williams DA, et al. Chloromethyl-Xrosamine (MitoTracker Red) photosensitizes mitochondria and induces apotosis in intact human cells. J Cell Sci 112: 2419-2430, 1999.

13. Kamada T, Koizumi T, Tsujii $\mathrm{M}$, et al. Clinical evaluation by double-blind trial of taurine for acute hepatitis. Sulfur-containing Amino Acid 3: 223-235, 1980 (in Japanese).

14. Pion PD, Kittleson MD, Rogers QR, et al. Myocardial failure in cats associated with low plasma taurine: a reversible cardiomyopathy. Science 237: 764-768, 1987.

(C) 2012 The Japanese Society of Internal Medicine http://www.naika.or.jp/imonline/index.html 